\title{
$S l_{q}(2)$ 无穷维表示相关的 $R$-矩阵及其 有限维分解结构*
}

\author{
孙昌璜 \\ （东北师范大学物理系，长春 130024） \\ 葛墨 林 \\ (南开数学研究所, 天津 300071)
}

\section{关链词 . 杨-Baxter 方程、量子代数、无穷维表示}

目前, 联系于量子代数 $u_{q}(\mathscr{L})$ 的有限维表示人们不仅得到了量子杨-Baxter 方程 (QYBE) 的标准解 $(R-\text { 矩阵) })^{[1-3]}$, 而且在 $q$ 为单位根情况下构造了各种新型 $R$ 矩阵(包括非 标准解和有颜色解 $)^{[4-7]}$. 本文将致力于构造另一类新的 $R$ 矩阵, 这类 $R$ 矩阵联系于量子代数 的无穷维表示. 由于采用的无穷维表示是不可约的, 得到的 $R$ 矩阵不能分解成通常 $R$ 矩阵的 直和; 相反, 它的有限维直和分解自动导致新型 $R$ 矩阵. 本文的讨论是以 $S l_{q}(2)$ 情况为例进 行的,但其中的基本思想和方法可以应用到其它量子代数.

本文的出发点是 $S l_{q}(2)$ Verma 空间 $\left.V^{n}:\left\{f_{2}(n)-J_{+}^{n}|\lambda\rangle\left|J_{-}\right| \lambda\right\rangle\left|-0, J_{3}\right| \lambda\right\rangle--\lambda|\lambda\rangle$, $\left.n \in \mathbf{Z}^{+}-\{0,1,2, \cdots\}\right\}$ 上的 Verma 表示 $\rho^{\wedge}$ :

$$
\left\{\begin{array}{l}
\rho^{\lambda}\left(J_{+}\right) f_{2}(n)-f_{\lambda}(n+1), \\
\rho^{\lambda}\left(J_{-}\right) f_{2}(n)-[n][\lambda+1-n] f_{\lambda}(n-1), \\
\rho^{\lambda}\left(J_{3}\right) f_{\lambda}(n)-(2 n-\lambda) f_{2}(n),
\end{array}\right.
$$

其中 $[n]-\left(q^{n}-q^{-n}\right) /\left(q-q^{-1}\right)$. 应用普适 $R$ 矩阵

$$
\mathscr{R}=\sum_{u} e_{a} \otimes e^{a}-q^{J_{3} \otimes J_{3} / 2} \sum_{n=0}^{\infty} \frac{\left(1-q^{-2}\right)^{n}}{[n] !} q^{\frac{1}{2} n(n-1)}\left(q^{J_{\alpha} / 2} J_{+} \otimes q^{-J_{3} / 2} J_{-}\right)^{n} .
$$

由上述表示 $\rho^{\lambda}$ 可确定无穷维 $R$ 矩阵 $R\left(\lambda \lambda^{\prime}\right)=\rho^{\lambda} \otimes \rho^{\lambda^{\prime}}(\mathscr{R}) \in \operatorname{End}\left(V^{\wedge} \otimes V^{\lambda}\right)$ 自动满足无谱参 数 QYBE:

$$
R_{12}\left(\lambda_{1}, \lambda_{2}\right) R_{13}\left(\lambda_{1}, \lambda_{3}\right) R_{23}\left(\lambda_{2}, \lambda_{3}\right)-R_{23}\left(\lambda_{2}, \lambda_{3}\right) R_{13}\left(\lambda_{1}, \lambda_{3}\right) R_{12}\left(\lambda_{1} \lambda_{2}\right),
$$

其中 $R_{i j}\left(\lambda_{i}, \lambda_{i}\right)$ 代表 $R\left(\lambda, \lambda^{\prime}\right)$ 作用于 $\operatorname{End}\left(V^{\lambda_{1}} \otimes V^{\lambda_{2}} \otimes V^{\lambda_{3}}\right)$ 中的第 $i$ 和 $i$ 分量上. 作用于 $V^{\lambda_{1}} \otimes V^{\lambda_{2}} \otimes V^{\lambda_{3}}$ 上的算子集 $\hat{O}:\left\{R_{12}\left(\lambda_{1}, \lambda_{2}\right), R_{13}\left(\lambda_{1}, \lambda_{3}\right), R_{23}\left(\lambda_{2}, \lambda_{3}\right)\right\}$ 使得基矢

$$
f\left(m_{1}, m_{2}, m_{3}\right)-f_{\lambda_{1}}\left(m_{1}\right) \otimes f_{\lambda_{2}}\left(m_{2}\right) \otimes f_{\lambda_{3}}\left(m_{3}\right),\left(m_{i} \in \mathbf{Z}^{+}, i-1,2,3\right)
$$

的指标和 $m_{1}+m_{2}+m_{3}$ 保持不变. 因而有一个

$$
d_{N}-\frac{(N+2) !}{2 ! N !}-\frac{1}{2}(N+2)(N+1)(\text { 维 }),
$$

不变子空间 $W^{N}=W^{N}\left(\lambda_{1}, \lambda_{2}, \lambda_{3}\right):\left\{f\left(m_{1}, m_{2}, m_{3}\right) \mid m_{1}+m_{2}+m_{3}=N\right\}\left(N \in Z^{+}\right)$. 把 $R_{i j}\left(\lambda_{i}\right.$, $\left.\lambda_{i}\right)$ 限制到 $W^{N}$ 上, 立即得到一个 $d_{N} \times d_{N}$ 的 $R$ 矩阵 $R_{i j}^{N}\left(\lambda_{i}, \lambda_{i}\right)$, 它自然满足 QYBE(3). 于

1992-03-16 收稿,1992-06-08 收修改稿

* 国家自然科学基金资助项目 
是我们得到 $R$ 矩阵在无穷维时的有限分解

$$
R_{i j}\left(\lambda_{i}, \lambda_{j}\right)-\bigoplus_{N=0}^{\infty} R_{i j}^{N}\left(\lambda_{i}, \lambda_{j}\right),
$$

其中每一个子块 $R_{i j}^{N}\left(\lambda_{i}, \lambda_{i}\right)$ 均满足 QYBE,其矩阵元由下式明显确定:

$$
\begin{aligned}
& \boldsymbol{R}_{i j}\left(\lambda_{i}, \lambda_{i}\right) f\left(m_{1}, m_{2}, m_{3}\right)-\sum_{m_{1}^{\prime}+m_{2}^{\prime}+m_{3}^{\prime}-N} R_{i j}^{N}\left(\lambda_{i}, \lambda_{i}\right)_{m_{i} m_{2} m_{2}}^{m_{1}^{\prime} m_{m}^{\prime} m_{3}^{\prime}} f\left(m_{1}^{\prime}, m_{2}^{\prime}, m_{3}^{\prime}\right) \\
& -\sum_{n=0}^{m i} \frac{1}{[n] !}\left(1-q^{-2}\right)^{n} q^{*\left(\frac{3}{2} n-\frac{3}{2}+\lambda_{j}-2 \lambda_{i}+2 m_{i}-m_{j}\right)^{*}} \\
& \text { - } q^{\frac{1}{2} n\left(2 m_{i}-2 m_{j}+\lambda_{j}-\lambda_{i}+3 n+1\right)} \cdot q^{\frac{1}{2}\left(m_{i}+2 n-\lambda_{i}\right)\left(2 m_{j}-2 n^{\left.-\lambda_{j}\right)}\right.} \\
& \text { - }\left\{\sum_{k=m i}^{m i-n+1}\left\{[k]\left[\lambda_{i}+1-k\right]\right\}\right\} \\
& \cdot f\left(m_{1}+n \delta_{i 1}, m_{2}+n \delta_{i 2}-n \delta_{i 2}, m_{3}-n \delta_{i 3}\right) \text {. }
\end{aligned}
$$

现在给出一个 $N=1$ 时的 3 维 $R$ 矩阵的例子

$$
\begin{aligned}
& R_{12}^{1}\left(\lambda_{1}, \lambda_{2}\right)-\left[\begin{array}{ccc}
a_{12} & b_{12} & 0 \\
0 & a_{12} & 0 \\
0 & 0 & c_{12}
\end{array}\right], R_{13}^{1}\left(\lambda_{1}, \lambda_{3}\right)-\left[\begin{array}{ccc}
a_{13} & 0 & b_{13} \\
0 & c_{13} & 0 \\
0 & 0 & a_{13}
\end{array}\right], \\
& \boldsymbol{R}_{23}^{1}\left(\lambda_{2}, \lambda_{3}\right)=\left[\begin{array}{ccc}
c_{23} & 0 & 0 \\
0 & a_{23} & b_{23} \\
0 & 0 & a_{23}
\end{array}\right],
\end{aligned}
$$

其中 $a_{i j}-q^{\frac{1}{2} \lambda_{j}\left(\lambda_{i}-2\right)}, b_{i j}-a_{i j}\left[\lambda_{j}\right]\left(1-q^{-2}\right) q^{\frac{1}{2}\left(2-\lambda_{i}+\lambda_{j}\right)}, c_{i j}-q^{\frac{1}{2} \lambda_{i} \lambda_{j}}$, 可以直接验证以上的 $R$ 矩阵 $R_{\mathrm{1}_{2}}\left(\lambda_{1}, \lambda_{2}\right) \cdots \cdots$ 的确满足 QYBE.

最后,我们分析上述 $R$ 矩可能的物理含义. 事实上, 普通的 $R$ 矩阵 $R=\left(R_{e f}^{e b}\right)$ 表征了三 体散射过程中有自旋粒子的二体因子化 $s$ 矩阵. 通常可以用平面上的辫子群操作 ${ }_{e}^{a}>_{f}^{b}{ }^{b}$ 来代 表它, 相应的因子化过程可由图 1 的两个辫子群图形的拓扑等价来代表. $R$ 矩阵的权守恒条 件 ${ }^{[9,10]}$,意味着两两碰撞时的自旋守恒—“两两自旋守恒 ${ }^{[1]}$ :
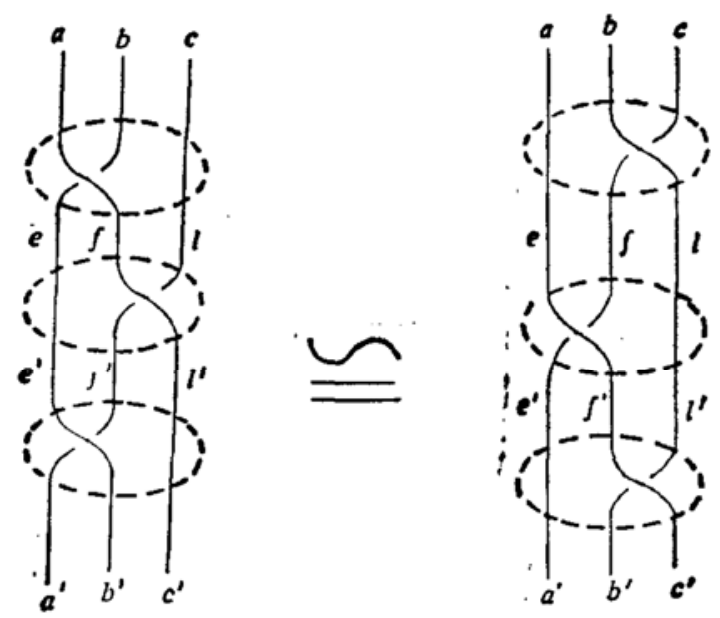

图 1 新型 $R$ 矩阵的图形表示

对于普通 $R$ 矩阵, 左图中 $e=e^{\prime}, c=l, l^{\prime} \cdots c^{\prime}$; 对于本文的 $R$ 矩阵, 这个限制消除 


$$
a+b=e+f, f+c-f^{\prime}+l^{\prime}, e+f^{\prime}-a^{\prime}+b^{\prime} .
$$

然而, 本文构造的 $R$ 矩阵 $R_{i j}^{N}\left(\lambda_{i}, \lambda_{i}\right)$ 仅要求三体碰撞的“出态”和“人态”的总自旋守恒:

$$
a+b+c-e+f+l-e^{\prime}+f^{\prime}+l^{\prime}=a^{\prime}+b^{\prime}+c^{\prime},
$$

而无需满足“两两自旋守恒”. 在图 1 中,我们用

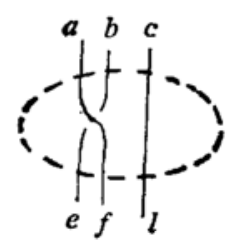

代表这样的 $R$-矩阵, 它对

总自旋 $N$ 确定的过程加以分类描述, 而无需区分个别粒子的自旋. 当然要把这样的 $R$ 矩阵联 系于实际的物理问题,还需要进一步的工作.

致谢作者感谢薛康、刘旭峰有益的讨论。

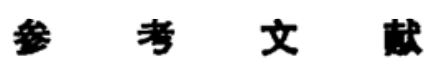

[ 1 ] Drifeld, V. G., Proc. IMC, Berkeley, 1986, 79.

[2] Jimbo, M., Lett. Math. Phys, 10(1985), 63; 11(1986), 247.

[ 3 ] Reshetikhin, N. Y., LOMI preprint, E4 \& E8, 1987.

[ 4 ] Sun, C. P. et. al., J. Phys., A24(1991), L 545.

[ 5 ] Sun, C. P. et. al., J. Moth. Phys, 32(1991), 2409.

[6] Ge, M. L. et. al., Phys. Lent, A155(1991), 137.

[7] Ge, M. L. et. al., Phys. Lett, A160(1991), 433.

[ 8 ] Sun, C. P. et al., J. Phys., Al23(1990), L1199.

[ 9 ] Sun, C. P. et al., J. Phys., A23(1990), L645.

[10] Li, Y. Q., et al., J. Phys., A24(1991), 3267.

[11] Yang, C. N. Ge, M. L. (eds.), Braid Group, Knot Theory and Statistical Mechanics, World Scientific, Singapore, 1990. 